\title{
Interkulturelle Kompetenz in Gruppen
}

\author{
Kathrin Kordon $\cdot$ Andrea Sanz $\cdot$ Jutta Überacker
}

Die Auseinandersetzung mit „Interkulturalität“ ist nicht neu. Auch diesem Heft gehen zwei Ausgaben voraus, die sich dem Thema widmen (,betrifft: Interkulturalität“ 1/2008; „Interkulturelle Trainings“ 2/2009). Mit den vorliegenden Beiträgen steigen wir in den Diskurs ein, der aktuell bleibt, gleichsam aber von einer ständigen Veränderung gekennzeichnet ist, wohl ein grundlegendes Spezifikum des Phänomens „Kultur“.

Mittlerweile wäre es höchst redundant, an dieser Stelle auf die gesellschaftspolitischen Zusammenhänge hinzuweisen, die die Relevanz des Themas für die Arbeitswelt herleiten. Das Nebeneinander, vielmehr noch die „Verschränkung“ unterschiedlicher Kulturen ist in nahezu allen Arbeitskontexten - längst nicht mehr nur in internationalen Konzernen - Arbeitsalltag geworden. Multikulturelle Teams, inderdisziplinäre Zusammenarbeit, funktionale und sprachliche Vielfalt sind ein vertrautes Bild geworden. Wiewohl der Diskurs zu Inter- Multi- und Transkulturalität voranschreitet, ist die vertiefende Auseinandersetzung mit diesen Phänomenen in der unmittelbaren Arbeitspraxis der Beschäftigten mit Berührungsängsten verbunden. Schon aus diesem Grund erschien es uns angebracht einen (weiteren) Versuch zu starten, theoretische Ansätze und Modelle mit reflektierter Praxis in Verbindung zu bringen.

$\mathrm{Zu}$ diesem Zweck haben wir sechs AutorInnen, allesamt professionelle BeraterInnen, eingeladen, sich mit folgenden zwei Ebenen interkultureller Kompetenz anhand konkreter Projekte näher auseinander zu setzen und eigene Modelle vorzustellen:

- Welche Kompetenz(en) brauchen OrganisationsberaterInnen/SupervisorInnen, um auf dem interkulturellen Terrain Fuß fassen bzw. bestehen zu können?

- Welche Kompetenz(en) brauchen interkulturelle Gruppen und Teams bzw. FunktionsträgerInnen in interkulturellen Arbeitskontexten, um Arbeitsfähigkeit herzustellen?

Gemäß dem Thema sind die vorliegenden Beiträge von einer Diversität durchdrungen. Das Spektrum umfasst die Formate Weiterbildung, Supervision, Coaching, Training und Organisationsentwicklung in Profit und Social Profit Feldern ebenso wie einen englischen Beitrag im Kontext einer intervisorischen Arbeitssitzung. Nicht zuletzt sind erfreulicherweise 3 Frauen und 3 Männer als AutorInnen tätig geworden.

Online publiziert: 23.07 .2011

(C) VS Verlag für Sozialwissenschaften 2011

A. Sanz $(\bowtie) \cdot$ K. Kordon $\cdot$ J. Überacker

Wien, Österreich

E-Mail:sanz@naschmarkt.co.at 
Zwei davon - Hubert Kuhn und Jutta Überacker - haben ihre theoretischen Wurzeln in der Gruppendynamik, deren politischer Anspruch seit jeher um die Integration von Unterschieden kreist. Die Balance zwischen dem Widerspruch von Differenzierung und Integration in Gruppe und Gesellschaft war der Ausgangspunkt gruppendynamischen Forschungsinteresses für den Begründer Kurt Lewin und seine Partner Benne, Bradford und Lippitt in den späten 1930er und frühen 1940er Jahren.

Kurt Lewin gründete 1945 nicht nur das Forschungszentrum für Gruppendynamik im MIT (Massachusetts Institute of Technology). Im selben Jahr wurde von ihm auch die „Commission on Community Interrelations“ (C.C.I.) in Leben gerufen, mit der Zielsetzung neue Lösungskonzepte für das Zusammenleben der verschiedenen Ethnien in den USA zu finden. Das zwei Jahre später von ihm und seinem Team angebotene Antirassimus-Seminar ging mit der Entdeckung des Aktionsforschungsprinzips und den wesentlichen Elementen der Trainingsgruppe als „Connecticut Experiment“ in die Geschichte der Gruppendynamik ein, worauf Hubert Kuhn in seinem Beitrag Interkulturelle Kompetenz und gruppendynamisches Training. Eine ,, mission impossible“ oder die Rückkehr zu den Wurzeln? näher eingeht. In weiterer Folge beleuchtet er anhand von drei konkreten Sequenzen aus gruppendynamischen Trainingsgruppen gängige Modelle aus der Gruppendynamik in Hinblick auf ihre Anwendbarkeit für interkulturelle Trainings. Im Zuge dessen stellt er ein von ihm entwickeltes Pendel-Modell der Gruppenentwicklung vor, welches sich besonders für multikulturell zusammengesetzte Gruppen zur Formulierung von Entwicklungszielen eignet.

Den Impuls für das erwähnte Antirassismus-Seminar gaben ansteigende rassistische Spannungen in den Städten der amerikanischen Ostküste, im Fokus standen die Beziehungen zwischen Afro-AmerikanerInnen und amerikanischen JüdInnen, welche am Arbeitsmarkt der Nachkriegszeit als die am stärksten benachteiligte Gruppe galt. Während in den frühen Jahren der NTL's „Race“ und „Gender“ im Vordergrund standen, wurden in der weiteren Entwicklung mehrere Dimensionen von Gruppenzugehörigkeit in die Untersuchung von Ausschlussdynamiken von Gruppen mit einbezogen. Auf diesem Weg etablierte sich die Gruppendynamik nicht nur im interkulturellen Feld, sondern auch im Feld des Diversity Managements, wo sich unsere Autorin und Mitherausgeberin Jutta Überacker verortet. Sie behandelt in ihrem Beitrag Aushandeln von Kulturunterschieden im 30-Miuten-Takt die kulturelle Öffnung an der Schnittstelle mit Kunden und Kundinnen am Beispiel einer österreichweit tätigen Social Profitorganisation. In ihrem Fallbeispiel beraten ausnahmslos autochtone ÖsterreicherInnen Personen, die zu 20-50 \% eine andere Muttersprache als Deutsch sprechen und verschiedener kultureller wie nationaler Herkunft sind, in rechtlichen Fragestellungen. Sie gewährt uns Einblick in eine Projektarchitektur von der Auftragsgestaltung mittels Feldbeobachtung über Diagnose, Design und Durchführung bis hin zur Auswertung. Dabei arbeitet sie den Stellenwert der eigenen Haltung und Bereitschaft, sich auf die TeilnehmerInnen einzulassen, vor dem Hintergrund ihrer gruppendynamischen Prägung anschaulich heraus.

Margret Steixner lebte über 8 Jahre mit ihrer Familie in verschiedenen afrikanischen Ländern und betrieb während dieser Zeit intensive Forschung im Bereich der interkulturellen Kompetenzentwicklung. Vor diesem Hintergrund nimmt es nicht weiter Wunder, dass sie den Kompetenzbegriff beruhend auf realen Erfahrungen, respektive dem eigenen Erleben, mit dem Vermögen zur (Selbst-)Reflexion und verfügbaren Wissen verknüpft, 
definiert. In ihrem Beitrag Interkulturelle Kompetenz - Beleuchtung eines Entwicklungsprozesses, Methoden und Prozesse der interkulturellen Kompetenzentwicklung im Training und Coaching stellt sie unterschiedliche Trainingsansätze nebeneinander. Eine dem Leser/der Leserin in Form eines Steckbriefs vorgestellte Frau namens Irene, 35 Jahre, Österreicherin fungiert als durchgängige ,Folie‘, um die Frage der interkulturellen Kompetenzentwicklung anhand der Methoden Training \& Coaching näher durchzuspielen.

Gerhard Liska setzt sich in seinem Beitrag Kulturdiverse Teams im Rahmen internationaler Organisationsentwicklung: Ein heuristisches Modell der Kulturübersteigerung aus transkultureller Perspektive mit Problematiken interkultureller Gruppen (Subeinheiten und Teams) im Kontext der Organisationsentwicklung auseinander. Er sieht das Finden von Gemeinsamkeiten in einer Gruppe, die unterschiedliche Wertehaltungen und mentale Modelle prägen, als eine mögliche Form der Problembearbeitung. Basierend auf dem Konzept der Transkulturalität, hat Liska ein 4-Stufen-Modell entwickelt, das den idealtypischen Verlauf von der Wahrnehmung der Unterschiede zur Etablierung einer Meta-Kultur veranschaulicht. Dieser Prozess setzt voraus, dass die wahrgenommenen Unterschiede überwunden werden und das „Gemeinsame“ auf der strukturell-organisatorischen Ebene in Form von konkreten Handlungslinien verankert wird.

Siegfried Tatschl widmet sich der Gesellschaftsdynamik der Interkulturalität und stellt seinen Implikationen für Supervision und Organisationsberatung die Beobachtung voran, dass Trainings und Weiterbildungen zum Thema „Migration und Interkulturalität“ nach wie vor Minderheitenprogramme sind, ganz im Gegensatz zu der Multikulturalität, die ihm in seiner Arbeit als Supervisor und Organisationsberater begegnen. Ihn beschäftigt die Frage warum Organisationen den Umstand, dass immer mehr Menschen mit Migrationshintergrund bei ihnen arbeiten, so zögerlich thematisieren. Er sieht es als Aufgabe von Beratung auf die veränderten Rahmenbedingungen und Referenzrahmen wie Seilschaften hinzuweisen. Für SupervisorInnen könnten Spiegelungsphänomene aus dem Kontext des Feldes dafür dienlich sein: ihrer Aufgabe wohne es ohnehin inne, vorübergehende Irritation und Desorientierung auszuhalten, dies führe häufig zu Anschlussängsten und der Sorge des Kompetenzverlustes, aggressiven Gefühlen und Selbstabwertung, die als Erfahrungen nutzbar gemacht werden können. Auch er plädiert hier für eine gezielte und intensive Auseinandersetzung/ Selbsterfahrung mit dem eigenen Zugehörigkeitserleben. Sein konzeptuelles Angebot zur kulturellen Öffnung von Institutionen lautet „Shifting Baselines“.

Kathrin Kordon, ebenfalls Mitherausgeberin des Heftes, bereichert den Diskurs durch Erkenntnisse aus der angewandten Sprachwissenschaft. Ihr interdisziplinärer Artikel Using English as a foreign language in international and multicultural consulting: Asset or Hindrance? kann gleichermaßen als Einladung gelesen werden, sich trotz vermeintlich fehlender oder unzureichender Sprachkenntnisse auf Beratungsformate in Englisch einzulassen. Sie analysiert eine Fallbearbeitung in einer interkulturellen Intervisionsgruppe deren TeilnehmerInnen allesamt Englisch als Zweit- oder Drittsprache sprechen, also Englisch als lingua franca (kurz ELF). Basierend auf ihren Ergebnissen, generiert sie die Hypothese, dass Supervisionen auf ELF trotz klarer Abweichungen von muttersprachlichen Normen erfolgreich verlaufen können und - darüber hinaus - die Verwendung von ELF positive Implikationen auf den Arbeitsprozess hat. 
Wir wünschen Ihnen eine spannende Lektüre und hoffen dass der interkulturelle Diskurs noch vielfach fortgesetzt wird, einen Beitrag dazu möge dieses Schwerpunktheft liefern. Für die Mitwirkung danken wir allen Autoren und Autorinnen, die ihre Zeit und Energie mit uns in dieses Projekt investiert haben.

Kathrin Kordon, Andrea Sanz, Jutta Überacker 